Check for updates

Cite this: RSC Adv., 2019, 9, 17841

Received 8th April 2019

Accepted 2nd June 2019

DOI: $10.1039 / c 9 r a 02610 j$

rsc.li/rsc-advances

\section{Activated carbons derived from hydrothermal impregnation of sucrose with phosphoric acid: remarkable adsorbents for sulfamethoxazole removal $\dagger$}

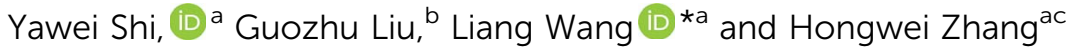

A series of activated carbons with surface areas of 925-1929 $\mathrm{m}^{2} \mathrm{~g}^{-1}$ were synthesized by in situ hydrothermal impregnation of sucrose with $\mathrm{H}_{3} \mathrm{PO}_{4}$ and subsequent calcination at $500-900{ }^{\circ} \mathrm{C}$. The prepared various types of activated carbons were utilized for the removal of sulfamethoxazole (SMX) from its solution by adsorption, and the effects of contact time, adsorbent dosage, initial concentration, adsorption temperature and $\mathrm{pH}$ on SMX adsorption were studied. The pseudo-second-order and the intra-particle diffusion model were used to analyze the adsorption kinetic data. The adsorption isotherm studies showed that the activated carbon prepared at $900{ }^{\circ} \mathrm{C}(\mathrm{C}-900)$ showed the highest Langmuir maximum adsorption capacity of $808.7 \mathrm{mg} \mathrm{g}^{-1}$ among them, much higher than that of C-500 $(274.0 \mathrm{mg}$ $\mathrm{g}^{-1}$ ). Adsorption thermodynamic results showed that the adsorption of SMX was a spontaneous exothermic process, with a standard enthalpy change of $-6.59 \mathrm{~kJ} \mathrm{~mol}^{-1}$ and a standard entropy change of $47.7 \mathrm{~J} \mathrm{~mol}^{-1} \mathrm{~K}^{-1}$. It was deduced that hydrophobic, electron donor-acceptor and electrostatic interactions were involved in the adsorption mechanism. Finally, regeneration experiments showed that more than $90 \%$ of the adsorption capacity could be recovered after four cycles through ethanol washing. Considering the remarkable and regenerable adsorption ability as well as the economic and environmental merits, these activated carbons are considered as promising candidates for potential practical applications in adsorptive removal of SMX.
\end{abstract}

\section{Introduction}

Antibiotics can enter the environment through sewage and improper disposal, threatening aquatic animals and human health. ${ }^{1}$ Sulfonamides are a class of synthetic antibiotics widely used for human and animal treatment due to their low price and wide spectrum antibiotic properties. ${ }^{2}$ Sulfonamides are stable in nature, not easy to be photolyzed or hydrolyzed, and easy to accumulate in water environments. ${ }^{3}$ As the most popular sulfonamide, sulfamethoxazole (SMX) has been detected quite frequently in both influent and effluent of worldwide waste water treatment plants, ${ }^{4}$ creating an urgent demand for its removal.

\footnotetext{
${ }^{a}$ State Key Laboratory of Separation Membranes and Membrane Processes, School of Environmental Science and Engineering, Tianjin Polytechnic University, Tianjin 300387, China. E-mail: mashi7822@163.com; Fax: +86 22 83955392; Tel: +86 22 83955392

${ }^{b}$ School of Chemical Engineering and Technology, Tianjin University, Tianjin 300072, China

${ }^{c}$ School of Environmental Science and Engineering, Tianjin University, Tianjin 300072, China

$\dagger$ Electronic supplementary information (ESI) available. See DOI: $10.1039 / \mathrm{c} 9 \mathrm{ra} 02610 \mathrm{j}$
}

Among various treatment technologies, adsorption is regarded to be a promising one due to it easy operation and absence of toxic byproducts in the process. ${ }^{5}$ Diversities of adsorbents have been investigated for SMX removal, and carbon materials with abundant porous structures have particularly gained attention. ${ }^{6}$ Some researchers have been focusing on the modifications of activated carbons through heteroatom doping or metal loading for better removal performances. ${ }^{7}$ Other researchers explored the possibilities of emerging carbon materials in this field, including ordered microporous carbons, ${ }^{8}$ graphene-based materials, ${ }^{\mathbf{9}}$ carbon nanotubes, ${ }^{\mathbf{1 0}}$ and metalorganic-framework-derived carbons. ${ }^{11,12}$

Recently, carbon materials synthesized by the hydrothermal carbonization (HTC) of carbohydrate biomass are attracting increasing attention. ${ }^{13,14}$ HTC is known as an economic approach due to the utilization of cheap biomass precursors and low preset carbonization temperatures. To develop porosity in the carbons produced by HTC, a post-treatment activation procedure with $\mathrm{KOH}, \mathrm{H}_{3} \mathrm{PO}_{4}$ or $\mathrm{ZnCl}_{2}$ is usually required. For instance, $\mathrm{KOH}$ has been employed for the generation of porosity in our previous work, ${ }^{\mathbf{1 5}}$ where the hydrothermal char from sucrose needs to be separated and dried after HTC, mixed with $\mathrm{KOH}$ by impregnation, then dried again, calcined, washed and 
dried for a third time to get the resulting activated ones. The whole activation process is effective, but tedious and timeconsuming.

As an alternative, an in situ approach known as hydrothermal impregnation has been proposed by Wisniewski et al. ${ }^{\mathbf{1 6}}$ In this approach, the whole preparation process could be simplified by mixing the carbohydrate precursor with $\mathrm{H}_{3} \mathrm{PO}_{4}$ before the HTC procedure. In the pioneer work of Wisniewski et al. and following works, ${ }^{\mathbf{1 6 - 1 8}}$ the calcination step was usually conducted at 400-800 ${ }^{\circ} \mathrm{C}$. Recently, Lei et al. ${ }^{19}$ employed glucose and phosphorus pentoxide $\left(\mathrm{P}_{2} \mathrm{O}_{5}\right)$ for the fabrication of porous carbon nanosheets. It was found that when the calcination temperature reached $900{ }^{\circ} \mathrm{C}$, and additional pores were introduced through redox reactions between carbon and $\mathrm{P}_{2} \mathrm{O}_{5}$. The dehydration of $\mathrm{H}_{3} \mathrm{PO}_{4}$ will lead to in situ production of $\mathrm{P}_{2} \mathrm{O}_{5} \cdot{ }^{20}$ We infer that increasing the activation temperature to $900{ }^{\circ} \mathrm{C}$ may help to activate the carbons better through the redox reactions between carbon and $\mathrm{P}_{2} \mathrm{O}_{5}$ and thus further extent the potential of the hydrothermal impregnation approach.

The activated carbons derived from the hydrothermal impregnation approach have several environmental and economic merits. These carbons have not been used for SMX adsorption in previous reports. To investigate whether they can be used as potential candidates for SMX adsorption, the effect of calcination temperature on the adsorption performance of the resulting activated carbon was studied in an extended range up to $900{ }^{\circ} \mathrm{C}$ in this work. The effects of contact time, adsorbent dosage, initial concentration, adsorption temperature and $\mathrm{pH}$ as well as the regeneration property were also investigated in detail. The discrepant adsorption performances of the carbons were related to the diversities in their textural and chemical properties, and the plausible adsorption mechanism was proposed as well.

\section{Experimental}

\subsection{Materials}

Sucrose (AR) and $\mathrm{H}_{3} \mathrm{PO}_{4}(85 \%)$ were obtained from Tianjin Fengchuan Chemical Reagents (Tianjin, China) and Tianjin Boute Chemical Reagents (Tianjin, China), respectively. Sulfamethoxazole (SMX, 98\%) was purchased Macklin Biochemical Co., Ltd (Shanghai, China). Milli-Q deionized water was used in all experiments.

\subsection{Preparation}

In a typical synthesis, sucrose and $\mathrm{H}_{3} \mathrm{PO}_{4}$ were dissolved to obtain a solution containing $40 \mathrm{~mL}$ water. The concentration of sucrose was $0.8 \mathrm{~mol} \mathrm{~L}^{-1}$, and the mass ratio of $\mathrm{H}_{3} \mathrm{PO}_{4}$ to sucrose was $1.7: 1$. Here the concentration of sucrose $\left(0.8 \mathrm{~mol} \mathrm{~L}^{-1}\right)$ was commonly used in previous reports for hydrothermal carbonization. The ratio of $1.7: 1$ was selected based on the report by Romero-Anaya et al. ${ }^{21}$ In that work, it was found that higher concentrations led to limited improvement in the final surface areas. The solution was put into a $100 \mathrm{~mL}$ Teflon-lined stainless steel autoclave and then heated at $180{ }^{\circ} \mathrm{C}$ in an oven for $8 \mathrm{~h}$. The resulting mixture was dried at $120{ }^{\circ} \mathrm{C}$ for $4 \mathrm{~h}$, calcined at $500-$
$900{ }^{\circ} \mathrm{C}$ for $2 \mathrm{~h}$ under nitrogen atmosphere $\left(30 \mathrm{~mL} \mathrm{~min}{ }^{-1}\right)$ in a horizontal quartz furnace tube (SK-G08125K, Tianjin Zhonghuan Corp., China), washed thoroughly with hot water, dried, crushed and sieved to select the fraction of 100-200 mesh. The resulting carbon prepared at the temperature of $x{ }^{\circ} \mathrm{C}$ was denoted as C- $x$.

\subsection{Characterization}

The activated carbons were characterized by scanning electron microscopy (SEM, S4800, Hitachi, Japan), transmission electron microscopy (TEM, G2 F20, Tecnai, USA), X-ray diffraction (XRD, D8 Advance Eco, Bruker, Germany) and Fourier transform infrared (FT-IR, iS50, Nicolet, USA). The samples were covered with gold as a conducting metal for SEM measurements. CHN elemental analyses were conducted on a cube instrument (Vario EL, Elementar Analyser systeme GmbH, Germany) to measure the contents of carbon and hydrogen, and the phosphorus contents were measured with inductively coupled plasmaatomic emission spectrometry (ICP-AES, ICP-9000, Thermo Jarrell-Ash Corp, USA). The oxygen contents were determined by difference. X-ray photoelectron spectroscopy (XPS) was conducted using a K-alpha spectrometer (Thermofisher, USA). Raman spectra were recorded on an XploRA Plus system (Horiba, Japan) at an excitation wavelength of $532 \mathrm{~nm}$. Zeta potential values were determined by Nano-ZS90 (Malvern Instruments, UK). The nitrogen sorption isotherms of the samples were recorded on Autosorb-iQ-C (Quantachrome, USA) after degassing at $290{ }^{\circ} \mathrm{C}$ for $10 \mathrm{~h}$. The specific surface areas were calculated by the Brunauer-Emmett-Teller (BET) method using the relative pressure $\left(p / p_{0}\right)$ ranges selected based on criteria established previously. ${ }^{22}$ For the pore size distributions, the nonlocal density functional theory (NL-DFT) method was used with the slit/cylinder model for nitrogen at $77 \mathrm{~K}$ on carbon, and the fitting errors were all below $0.5 \%$. The total pore volumes were obtained from the points at saturated pressures on the isotherms.

\subsection{Adsorption experiments}

The SMX solution (100 $\mathrm{mg} \mathrm{L}^{-1}$ ) was prepared by dissolving SMX in water without co-solvent. The adsorption experiments were conducted in a shaker bath with a rotation speed of $180 \mathrm{rpm}$ at $30{ }^{\circ} \mathrm{C}$ unless otherwise stated. Kinetic studies were performed by adding $10 \mathrm{mg}$ activated carbon to $50 \mathrm{~mL} \mathrm{SMX} \mathrm{solution} \mathrm{with}$ an initial concentration of $50 \mathrm{mg} \mathrm{L}^{-1}$ at $\mathrm{pH} 5.0 \pm 0.1$. After an appropriate time, the solution was immediately separated from the activated carbon with a syringe filter (nylon, $0.22 \mu \mathrm{m}$ ), and the concentration of SMX was measured with a Cary 60 UVvisible spectrometer (Agilent) at $267 \mathrm{~nm}$. To investigate the effect of adsorbent dosages, a series of dosages $\left(0.05-0.3 \mathrm{~g} \mathrm{~L}^{-1}\right)$ were utilized at the same initial concentration $\left(50 \mathrm{mg} \mathrm{L}^{-1}\right)$ and $\mathrm{pH}(5.0 \pm 0.1)$. For isotherm fitting, an adsorbent dosage of $0.05 \mathrm{~g} \mathrm{~L} \mathrm{~L}^{-1}$ and a series of initial SMX concentrations (20$\left.100 \mathrm{mg} \mathrm{L}^{-1}\right)$ were used at $\mathrm{pH}(5.0 \pm 0.1)$. For thermodynamic study, additional adsorption experiments were conducted at 20 and $40{ }^{\circ} \mathrm{C}$ with the optimal adsorbent (C-900) at $\mathrm{pH}(5.0 \pm 0.1)$. It should be noted that although the absorption spectrum of 
SMX changes with $\mathrm{pH}$, the change in $\mathrm{pH}$ was found to be less than 0.1 when the initial $\mathrm{pH}$ was around 5.0. Thus the wavelength for UV-vis measurements in the above tests was fixed at $267 \mathrm{~nm}$ using a standard curve built at $\mathrm{pH}$ 5.0. To investigate the effect of $\mathrm{pH}$ value on adsorption, SMX solutions $\left(50 \mathrm{mg} \mathrm{\textrm {L } ^ { - 1 }}\right)$ with different $\mathrm{pH}$ values adjusted by $\mathrm{HCl}$ or $\mathrm{NaOH}$ were treated with $0.2 \mathrm{~g} \mathrm{~L}^{-1} \mathrm{C}-900$. The solution after adsorption was separated from the adsorbent and then diluted with a $\mathrm{HCl}$ or $\mathrm{NaOH}$ solution to adjust the $\mathrm{pH}$ to around 5.0. The concentration after dilution was then measured by UV-vis at $267 \mathrm{~nm}$, and the concentration before dilution was calculated based on the dilution ratio. The recyclability of C-900 for SMX adsorption was investigated. After the first adsorption run, the spent adsorbent was separated and then stirred with ethanol for $2 \mathrm{~h}$. After that, the adsorbent was separated again for the next adsorption run. The amount of ethanol used was $50 \mathrm{~mL}$ per $10 \mathrm{mg}$ fresh adsorbent. When $10 \mathrm{mg}$ activated carbon was used, the recovery rate was only around $60 \%$. This was attributed to the mechanical loss during the regeneration process, such as the attachment of carbon to the stirrer and the filtration membrane. When a larger amount of carbon (60 mg) was regenerated, the recovery rate was increased to $94 \%$. Thus, spent adsorbents from parallel adsorption experiments were mixed together, but the dosage for adsorption was fixed at $0.2 \mathrm{~g} \mathrm{~L}^{-1}$.

\subsection{LCA assessment}

The life cycle assessment (LCA) analysis ${ }^{23,24}$ is a useful tool for the evaluation of environmental impacts of the activated carbon. The cradle-to-grave scheme of the carbon has been provided in Fig. S1 in ESI. $\dagger$ The scheme describes the input and output during the carbon preparation procedure, which helps to determine the system boundaries for the LCA analysis. Further research is needed to conduct a full LCA analysis of the carbon.

\section{Result and discussion}

\subsection{Characterizations of the activated carbons}

As shown in Fig. 1a-e, the activated carbons were generally spherical in shape, in accordance with previous reports. ${ }^{\mathbf{1 6}}$ TEM analysis of C-900 was further conducted. As illustrated in Fig. S2a and $b, \dagger$ the carbon was spherically shaped and several micrometers in diameter, in consistent with SEM results. The pores in bulk were difficult to be viewed in TEM large due to the large thickness of the carbon spheres, but irregular pores could be observed in the small fragments (Fig. S2c $\dagger$ ) and at the borders of the sphere (Fig. S2d †), verifying its porous structure. XRD patterns (Fig. 1f) of the activated carbons revealed their amorphous nature, showing two broad bands at around $24^{\circ}$ and $43^{\circ}$ corresponding to (002) and (10) diffraction peaks. ${ }^{25}$ With an increase in the activation temperature, the peaks shifted slightly to a smaller angle, indicating an increased layer-to-layer distance. This may be attributed to the increased defects created during the activation process. ${ }^{26}$

The nitrogen sorption isotherms (Fig. 2a) are mixtures of type I and type IV with hysteresis loops, indicating the hierarchical porous structures in the carbons. Both the specific surface areas and the pore volumes tended to be promoted with the rise in the activation temperature (Table 1), and the pore size distributions tended to shift to larger pore sizes (Fig. 2b).

During the activation process, the decomposition of $\mathrm{H}_{3} \mathrm{PO}_{4}$ resulted in the formation of $\mathrm{P}_{2} \mathrm{O}_{5}$ and water. ${ }^{20}$ The sublimation of $\mathrm{P}_{2} \mathrm{O}_{5}$ was responsible for the generation of pores. Water molecules also supported the development of porosity through carbon gasification (eqn (1)). With an increase in the calcination temperature, these endothermic reactions became stronger, leading to more porosity in the resulting carbon. This is also reflected in the declining carbon yields at higher temperatures (Table 1). When the temperature of $900{ }^{\circ} \mathrm{C}$ was utilized, the surface area was boosted dramatically to $1929 \mathrm{~m}^{2} \mathrm{~g}^{-1}$. At this temperature, $\mathrm{P}_{2} \mathrm{O}_{5}$ created by the decomposition of $\mathrm{H}_{3} \mathrm{PO}_{4}$ can act as an oxidation agent through eqn (2), which is a highly endothermic reaction and occurs at around $900{ }^{\circ} \mathrm{C} .{ }^{19}$ Through this reaction, the etching of the carbon framework led to the creation of more pores, and the yield decreased further. We tried to promote the activation process by employing a higher temperature of $1000{ }^{\circ} \mathrm{C}$. However, very little solid product with a yield below $1 \%$ was obtained, suggesting the almost complete consumption of carbon by eqn (2).

$$
\begin{gathered}
\mathrm{C}+\mathrm{H}_{2} \mathrm{O}=\mathrm{CO}+\mathrm{H}_{2} \\
\mathrm{P}_{2} \mathrm{O}_{5}+5 \mathrm{C}=2 \mathrm{P}+5 \mathrm{CO}
\end{gathered}
$$

The elemental compositions of the activated carbons were displayed in Fig. 2c. Generally, the heteroatom contents decreased with increasing temperatures, which was ascribed to the more severe decomposition of heteroatom functionalities at higher temperatures. ${ }^{27}$ FT-IR measurements were further conducted to investigate the types of surface functionalities. As shown in Fig. 2d, a broad band centered at around $3400 \mathrm{~cm}^{-1}$ was assigned to $\mathrm{O}-\mathrm{H}$ vibration in water. Two small bands at 2925 and $2850 \mathrm{~cm}^{-1}$ were attributed to $\mathrm{C}-\mathrm{H}$ stretching vibrations, and the absorption at $1580 \mathrm{~cm}^{-1}$ was assigned to $\mathrm{C}=\mathrm{C}$ vibration. Moreover, the bands at around 1633, 1405 and 1000$1200 \mathrm{~cm}^{-1}$ were attributed to $\mathrm{C}=\mathrm{O}, \mathrm{C}-\mathrm{P}$ and $\mathrm{C}-\mathrm{O}$ vibrations respectively ${ }^{28}$ revealing the presence of oxygen and phosphorus functionalities on the activated carbons, consistent with the elemental analysis results.

\subsection{Effect of contact time}

The effect of contact time on SMX adsorption was investigated in the time range of $15 \mathrm{~min}-48 \mathrm{~h}$. The results were illustrated in Fig. 3a, and the experimental data were fitted to the pseudofirst-order (eqn (3)) and the pseudo-second-order (eqn (4)) kinetic models.

$$
\begin{aligned}
& q_{t}=q_{\mathrm{e}}\left(1-\exp \left(-k_{1} t\right)\right) \\
& q_{t}=q_{\mathrm{e}}{ }^{2} k_{2} t /\left(1+q_{\mathrm{e}} k_{2} t\right)
\end{aligned}
$$

In the equations, $q_{\mathrm{e}}\left(\mathrm{mg} \mathrm{g}^{-1}\right)$ is the amount of SMX adsorbed at equilibrium; $q_{t}\left(\mathrm{mg} \mathrm{g}^{-1}\right)$ is the amount of SMX adsorbed at 

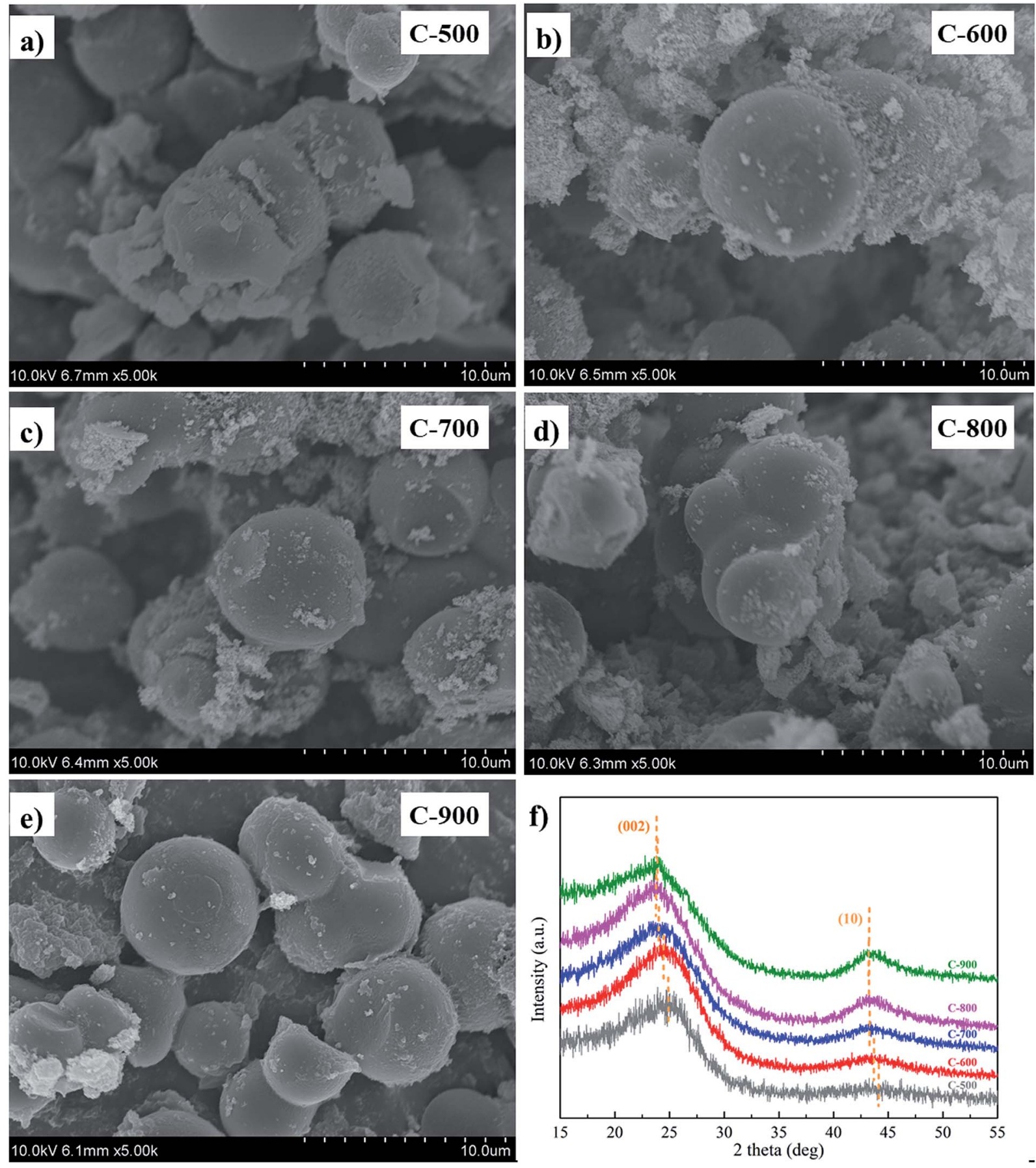

Fig. 1 SEM images (a-e) and XRD patterns (f) of the activated carbons.

the contact time of $t(\mathrm{~min}) ; k_{1}\left(\mathrm{~min}^{-1}\right)$ and $k_{2}\left(\mathrm{~g}\left(\mathrm{mg}^{-1} \mathrm{~min}^{-1}\right)\right)$ are the rate constants for the pseudo-fist-order and pseudosecond-order models, respectively. The fitting parameters derived by non-linear fitting and the initial adsorption rates $(h)$ calculated by eqn $(5)^{29}$ were listed in Table S1. $\dagger$

$$
h=k_{2} q_{\mathrm{e}}^{2}
$$

As compared in Table $\mathrm{S} 1, \uparrow$ the higher regression coefficient $\left(R^{2}\right)$ values suggested that the adsorption process matched better to the pseudo-second-order model. Besides, the calculated equilibrium adsorption amounts from the pseudosecond-order model were also closer to the expected values. Some researchers reported that the better fitting in the pseudosecond-order model indicated that the adsorption rate is mainly controlled by chemical sorption, while some others demonstrated that the adsorption mechanisms cannot be directly assigned based on fitting kinetic models. ${ }^{29}$ Despite of this controversy, a higher $h$ value in $\mathrm{mg} \mathrm{g}^{-1} \mathrm{~min}^{-1}$ should indicate a faster adsorption of SMX on the adsorbent. In this work, $h$ values increased with increasing calcination temperatures (Table S1 $\dagger$ ), in accordance with the order in mesopore volumes 

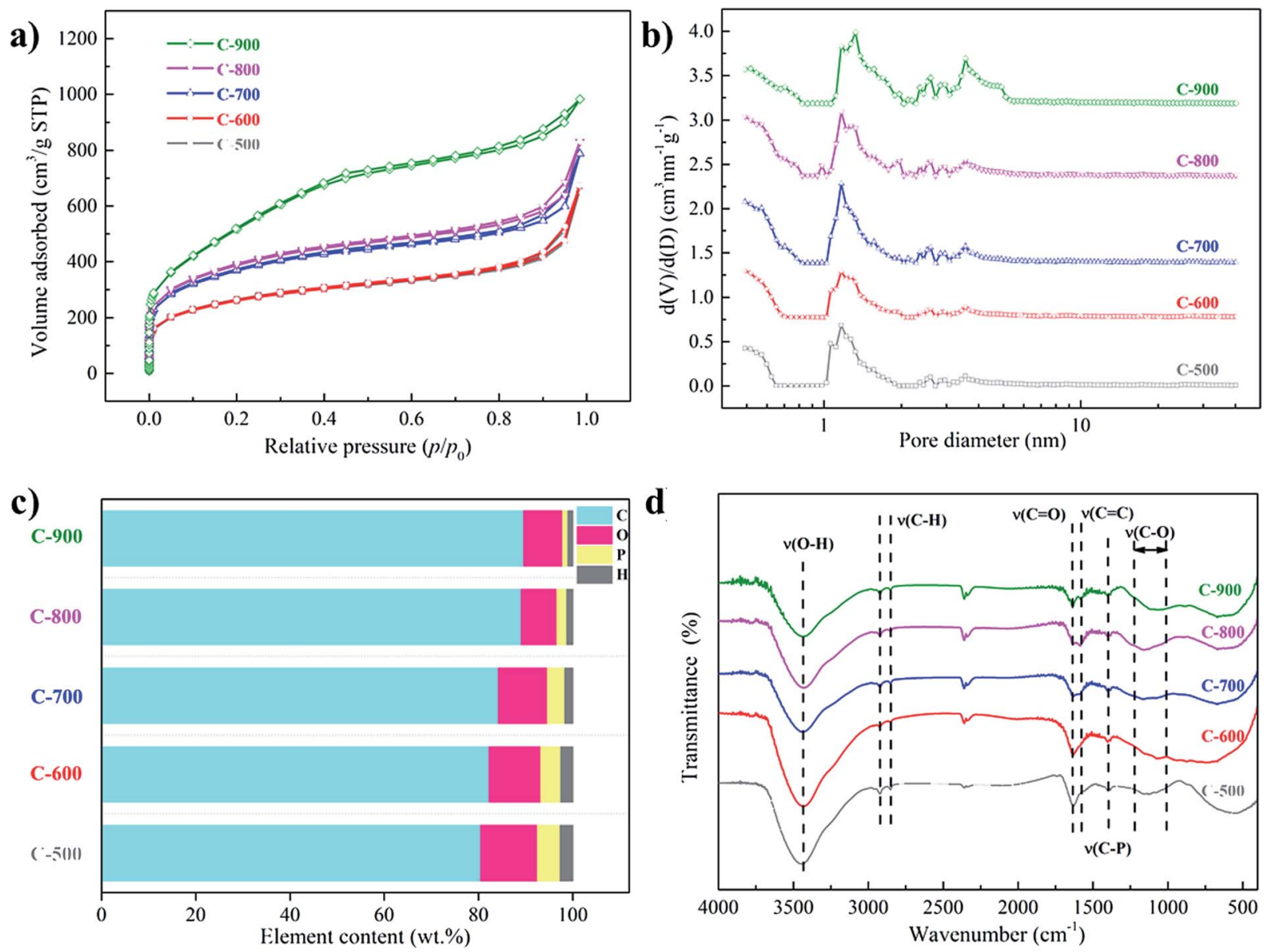

Fig. 2 Nitrogen sorption isotherms (a), pore size distributions (b), elemental contents (c) and FT-IR spectra (d) of the activated carbons.

$\left(V_{>2} \mathrm{~nm}\right)$ shown in Table 1. It is inferred that the presence of more mesopores in the carbon adsorbents could favor the diffusion of SMX, leading to faster adsorption rates. The kinetic date were also fitted in the intra-particle diffusion model..$^{30}$ The multi-linear curves (Fig. 3b) indicated that the whole adsorption process is controlled by a multistep mechanism including film diffusion as well as diffusion in the hierarchical porous structure, and the fall in rate constants with time $\left(k_{1}>k_{2}>k_{3}\right.$, Table S2 $\dagger$ ) is attributed to the gradual saturation of the adsorbent surface. $^{30}$

\subsection{Effect of adsorbent dosage and initial concentration}

The adsorption behaviors of SMX on the carbons were further evaluated under different adsorbent dosages. As illustrated in

Table 1 Textural properties and yields of the carbon adsorbents

\begin{tabular}{llllr}
\hline Sample & $S_{\text {BET }}{ }^{a}$ & $V_{\text {total }}{ }^{b}$ & $V^{b}{ }^{b} \mathrm{~nm}$ & $\begin{array}{l}\text { Yield } \\
(\%)\end{array}$ \\
\hline C-500 & 927 & 1.010 & 0.705 & 30.3 \\
C-600 & 925 & 1.042 & 0.747 & 27.5 \\
C-700 & 1301 & 1.219 & 0.788 & 22.4 \\
C-800 & 1368 & 1.286 & 0.870 & 13.4 \\
C-900 & 1929 & 1.521 & 1.102 & 8.8
\end{tabular}

${ }^{a} \mathrm{~m}^{2} \mathrm{~g}^{-1} \cdot{ }^{b} \mathrm{~cm}^{3} \mathrm{~g}^{-1}$.
Fig. 4a, a larger adsorbent dosage resulted in a lower adsorption amount for all the carbons, which could be attributed to the availability of more adsorption sites at higher dosages. ${ }^{15}$ The effect of initial concentration was also investigated. As expected, an increase in the adsorption amount could be observed with a higher initial concentration, and saturation has been reached for the carbons except for C-900 in the investigated concentration range (Fig. 4b). The experimental data were fitted to the Langmuir (eqn (6)) and Freundlich (eqn (7)) models ${ }^{31}$ by nonlinear fitting.

$$
\begin{gathered}
q_{\mathrm{e}}=q_{\max } K_{\mathrm{L}} C_{\mathrm{e}} /\left(1+C_{\mathrm{e}} K_{\mathrm{L}}\right) \\
q_{\mathrm{e}}=K_{\mathrm{F}} C_{\mathrm{e}}{ }^{1 / n}
\end{gathered}
$$

In the equations, $K_{\mathrm{L}}\left(\mathrm{L} \mathrm{mg}^{-1}\right)$ and $q_{\max }\left(\mathrm{mg} \mathrm{g}^{-1}\right)$ represent the Langmuir constant and the maximum adsorption capacity respectively, while $K_{\mathrm{F}}\left(\left(\mathrm{mg} \mathrm{g}^{-1}\right)\left(\mathrm{L} \mathrm{mg}^{-1}\right)^{1 / n}\right)$ and $n$ are Freundlich constants. The fitting parameters were shown in Table 2. The high regression coefficients indicated that both models could describe the adsorption behavior of SMX on the carbons. The calculated maximum adsorption capacities for C-500 and C-600 were close to each other due to the overlap of confidence intervals. The values of $K_{\mathrm{F}}$ representing adsorption capacities were also similar for C-500 and C-600, proving the credence of 
a)

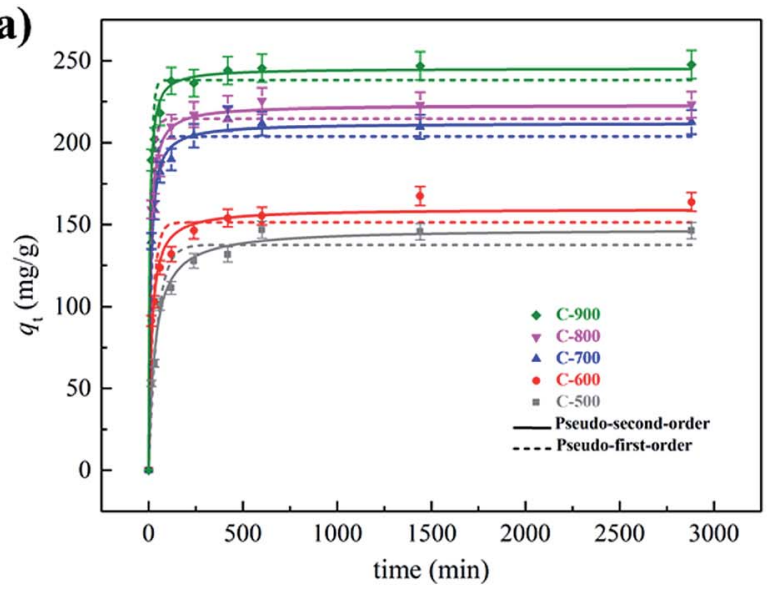

b)

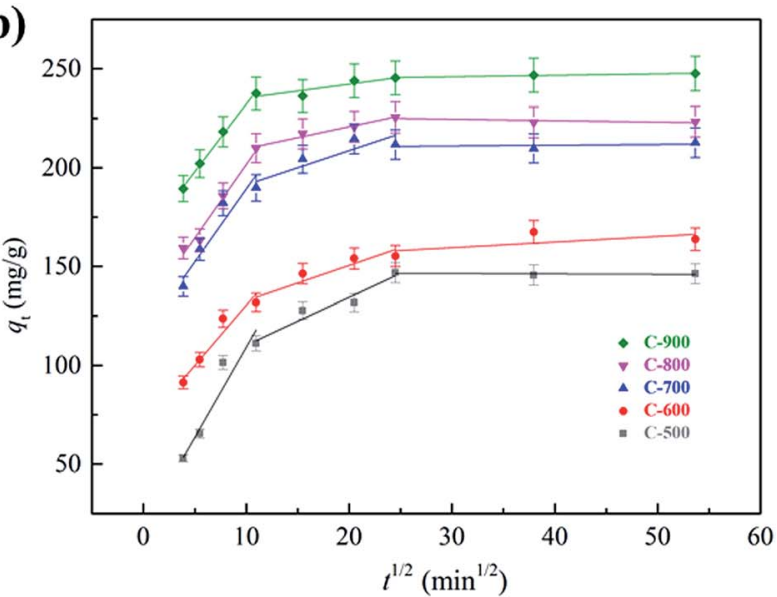

Fig. 3 Kinetic studies for SMX adsorption on the activated carbons: fitting in pseudo-first-order and pseudo-second-order models (a); fitting in the intra-particle diffusion model (b).

a)

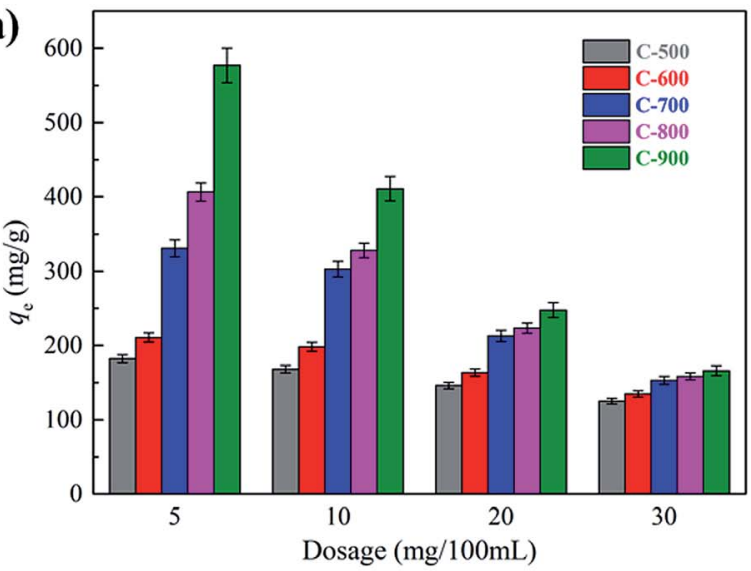

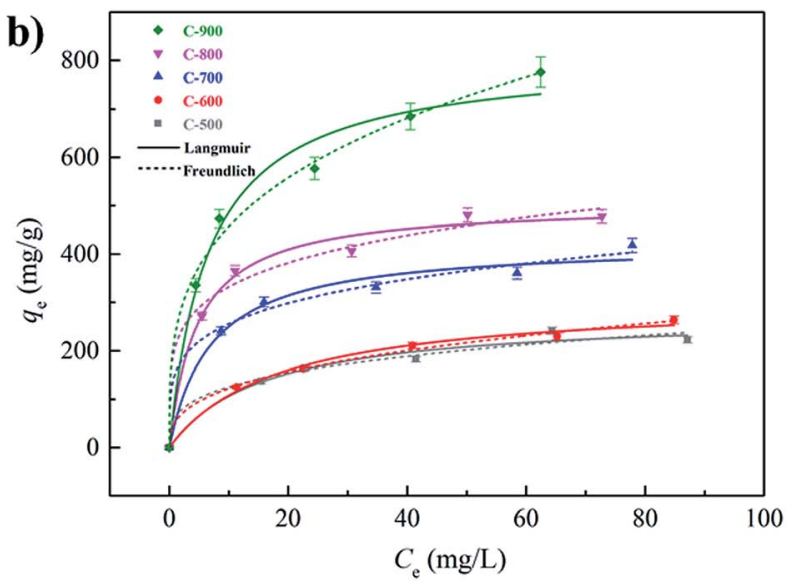

Fig. 4 Effect of adsorbent dosage (a) and initial concentration (b).

the results. For other samples, it could be found that higher calcination temperatures helped to promote the adsorption of SMX. The different adsorption capacities on the carbons were mainly attributed to their diversities in textural properties (see Section 3.6).

\subsection{Effect of adsorption temperature}

Taking the optimum carbon (C-900) as an example, the effect of adsorption temperature was studied furthermore. The maximum adsorption capacities of SMX were calculated to be
1142.7, 808.7 and $633.8 \mathrm{mg} \mathrm{g}^{-1}$ at 293,303 and $313 \mathrm{~K}$, respectively (Fig. S3 and Table S3†). The Gibbs free energy change $\left(\Delta G^{\circ}\right)$ was calculated by eqn (8).

$$
\Delta G^{\circ}=-R T \ln K_{\mathrm{c}}
$$

Here the equilibrium constant $\left(K_{\mathrm{c}}\right.$, dimensionless) was obtained from the distribution coefficient $\left(K_{\mathrm{d}}, \mathrm{L} \mathrm{g}^{-1}\right)$, and $K_{\mathrm{d}}$ was derived by plotting $\ln \left(q_{\mathrm{e}} / C_{\mathrm{e}}\right)$ against $C_{\mathrm{e}}$ and extrapolating to zero $C_{\mathrm{e}}{ }^{29}$ The standard enthalpy and entropy change $\left(\Delta H^{\circ}\right.$ and $\left.\Delta S^{\circ}\right)$ were obtained by fitting in the van't Hoff equation (eqn (9)).

Table 2 Isotherm models for SMX adsorption on the adsorbents

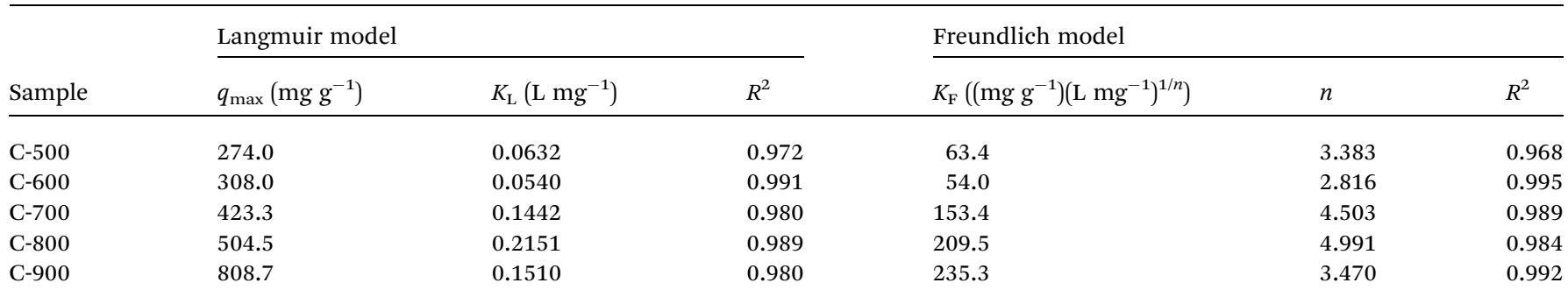


Table 3 Adsorption thermodynamics for SMX adsorption on C-900

\begin{tabular}{lllll}
\hline $\begin{array}{l}\text { Temperature } \\
(\mathrm{K})\end{array}$ & $K_{\mathrm{c}}$ & $\begin{array}{l}\Delta G^{\circ} \\
\left(\mathrm{kJ} \mathrm{mol}^{-1}\right)\end{array}$ & $\begin{array}{l}\Delta H^{\circ} \\
\left(\mathrm{kJ} \mathrm{mol}^{-1}\right)\end{array}$ & $\begin{array}{l}\Delta S^{\circ} \\
\left(\mathrm{J} \mathrm{mol}^{-1} \mathrm{~K}^{-1}\right)\end{array}$ \\
\hline 293 & 4637 & -20.57 & -6.59 & 47.70 \\
303 & 4235 & -21.04 & & \\
313 & 3902 & -21.52 & &
\end{tabular}

$$
\ln \left(K_{\mathrm{c}}\right)=-\Delta H^{\circ} / R T+\Delta S^{\circ} / R
$$

As shown in Table 3, the negative $\Delta G^{\circ}$ values indicated that the adsorption of SMX on the carbon was a spontaneous process. The negative $\Delta H^{\circ}$ value indicated the exothermic nature of SMX adsorption, which is in accordance with the decreasing $q_{\max }$ with increasing adsorption temperatures. It has been reported that ${ }^{32}$ the energies associated with hydrophobic interaction and EDA interaction were in the range of $0.4-4$ and $4-167 \mathrm{~kJ} \mathrm{~mol}^{-1}$. The relatively low enthalpy change indicated that adsorption of SMX on the carbon in this work was not firm. The transport of SMX from the aqueous phase to the solid adsorbent resulted in loss of freedom and thus decreased entropy, while the desorption of more than one water molecules which occurred simultaneously ${ }^{33}$ and the changes of the adsorbent surface ${ }^{34}$ led to increased entropy. The positive $\Delta S^{\circ}$ indicated the net increase of disorder during the whole adsorption process.

\subsection{Effect of $\mathrm{pH}$ value}

The adsorption behavior of SMX on C-900 was investigated under different $\mathrm{pH}$ values. As displayed in Fig. 5a, the adsorption amount of SMX was clearly $\mathrm{pH}$-dependent.

SMX is an amphoteric molecule with two $\mathrm{p} K_{\mathrm{a}}$ values of 1.6 (base) and 5.7 (acid). ${ }^{11}$ Thus, the dominate species at intermediate $\mathrm{pH}$ values are neutral molecules. ${ }^{32}$ The fraction of neutral molecules does not remain the same in the whole intermediate $\mathrm{pH}$ range (1.6-5.7). According to previous calculations, ${ }^{35}$ the fraction of neutral species $\left(\mathrm{SMX}^{0}\right)$ reaches almost $100 \%$ at around $\mathrm{pH}$ 3.7. From $\mathrm{pH} 3.7$ to $\mathrm{pH} 1.7$, the fraction of $\mathrm{SMX}^{0}$ decreases gradually to $50 \%$ while the fraction of positive species $\left(\mathrm{SMX}^{+}\right)$increases to $50 \%$. Similarly, from $\mathrm{pH} 3.7$ to $\mathrm{pH} 5.7, \mathrm{SMX}^{0}$ fraction drops while the fraction of negative species $\left(\mathrm{SMX}^{-}\right)$ rises to $50 \% . \mathrm{SMX}^{+}$and $\mathrm{SMX}^{-}$are the dominant species at $\mathrm{pH}<$ 1.6 and $\mathrm{pH}>5.7$, respectively. According to zeta-potential measurements (Fig. 5b), the isoelectric point (IEP) of C-900 was determined to be 5.4. Thus, the whole $\mathrm{pH}$ range could be divided into three zones: $\mathrm{pH}<3.7,3.7<\mathrm{pH}<5.4$, and $\mathrm{pH}>5.4$. At $\mathrm{pH}<3.7$, both $\mathrm{SMX}^{0}$ and $\mathrm{SMX}^{+}$were presented, and the surface of C-900 was positively charged. Therefore, electrostatic repulsion occurred between $\mathrm{SMX}^{+}$and $\mathrm{C}-900$. At $3.7<\mathrm{pH}<5.4$, electrostatic attraction existed between $\mathrm{SMX}^{-}$and the positively charged surface of C-900. Finally, electrostatic repulsion occurred again between $\mathrm{SMX}^{-}$and the negatively charged C-900 surface at $\mathrm{pH}>5.4$.

The change in electrostatic interactions with altering $\mathrm{pH}$ values could help to explain the trend in SMX adsorption amounts to some extent. However, as discussed above, if only electrostatic repulsion occurred at $\mathrm{pH}<3.7$ and $\mathrm{pH}>5.4$, the adsorption of SMX should be very low, which was against the result in Fig. 5a. Consequently, it was inferred that other adsorption mechanisms were also involved in the adsorption process.

\subsection{Adsorption mechanism}

Generally, textural properties of the adsorbents have an important impact on the adsorption process. As illustrated in Fig. S4a, $\dagger$ a good positive linear trend $\left(R^{2}=0.951\right)$ was observed between $q_{\text {max }}$ and $S_{\text {BET }}$. Based on previous works ${ }^{32,34,36}$ and this result, it was deduced that hydrophobic and electron donoracceptor (EDA) interactions were involved for SMX adsorption. A larger surface area provided more space for the interaction between SMX and the carbon surface, leading to a greater adsorption amount. The intensity of these interactions also changes with pH. Firstly, the greatest intensity of hydrophobic adsorption is expected when $\mathrm{SMX}^{0}$ is dominant because the neutral species exhibits the lowest solubility. ${ }^{9}$ Secondly, from the viewpoint of EDA interaction, SMX has a strong $\pi$-electron acceptor character whereas the carbon acts as a $\pi$-electron donor. When $\mathrm{SMX}^{0}$ is transformed into $\mathrm{SMX}^{+}$, the $\pi$-electron withdrawing ability is enhanced due to the protonation of the amino group. Conversely, the ability is weakened with the deprotonation of the sulfonamide group when $\mathrm{SMX}^{0}$ is
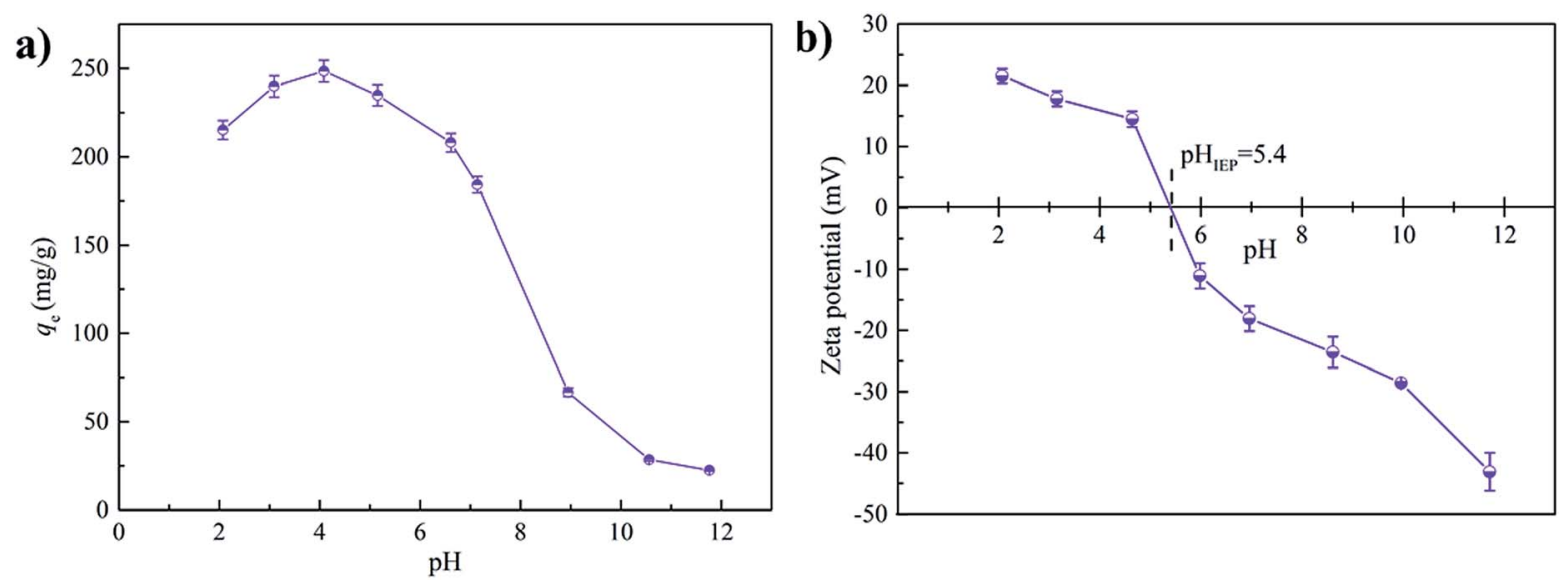

Fig. 5 Effect of pH on SMX adsorption amount (a) and zeta-potential of C-900 (b). 
Table 4 Plausible adsorption mechanisms at different $\mathrm{pH}$ ranges ${ }^{a}$

\begin{tabular}{llll}
\hline & $\begin{array}{l}\text { Hydrophobic } \\
\text { interaction }\end{array}$ & $\begin{array}{l}\text { EDA } \\
\text { interaction }\end{array}$ & $\begin{array}{l}\text { Electrostatic } \\
\text { interaction }\end{array}$ \\
\hline $\mathrm{pH}<3.7$ & $++^{b}$ & +++ & Repulsion \\
$3.7<\mathrm{pH}<5.4$ & +++ & ++ & $\begin{array}{l}\text { Attraction } \\
\text { Repulsion }\end{array}$
\end{tabular}

${ }^{a}$ The relative intensity is indicated by the number of plus signs. ${ }^{b}$ The solubility of $\mathrm{SMX}^{-}$is much larger than $\mathrm{SMX}^{+}$.

transformed into $\mathrm{SMX}^{-}$. Thus, the intensity of EDA interaction decreased with increasing $\mathrm{pH}$ values. Based on the above discussion, the plausible adsorption mechanisms involved in SMX adsorption at different $\mathrm{pH}$ ranges were summarized in Table 4 . Now the trend in Fig. 5a can be clearly understood.

It should be noted that other mechanisms ${ }^{\mathbf{1 1 , 3 5}}$ relating to the heteroatom functionalities on the carbon were considered as important driving forces for SMX adsorption in some previous reports. The activated carbons in this work also possessed heteroatom functionalities as discussed above, and their effects were evaluated by normalizing $q_{\max }$ for $S_{\text {BET }}$. By plotting $q_{\max } /$ $S_{\mathrm{BET}}$ against the heteroatom contents, a negative linear trend could be observed (Fig. S4b†), but the confidence intervals of some points overlapped and the correlation was weak with a low correlation coefficient. Firstly, the hydrophilic functionalities could weaken the hydrophobicity of the carbon surface and cause the formation of water clusters, ${ }^{37}$ which was detrimental for the hydrophobic adsorption of SMX. Secondly, the functionalities could also influence the strength of EDA interaction. Recent theoretical calculations ${ }^{38}$ revealed that different types of oxygen-containing functionalities could change the $\pi$ electron density of the carbon surface and thus affect the EDA interaction in different degrees. When it comes to phosphorus, the dopants in the carbon matrix acting as electron donors could increase the electron density of carbon ${ }^{39}$ and probably promote the EDA interaction as a result. Finally, hydrogen bonding ${ }^{\mathbf{1 1 , 3 5}}$ and surface reactive adsorption induced by the oxygencontaining groups ${ }^{36}$ may also make a contribution to the adsorption of SMX. In summary, the overall impact of heteroatoms on adsorption is convoluted and found to be slightly negative in this report due to the diversities in types of functionalities and their multiple impacts on the adsorption process.

To provide some experimental evidence for the above mechanism analysis, XPS and Raman measurements of a fresh C-900 sample and three spent samples after adsorption of SMX at $\mathrm{pH} 2,5$ or 9 respectively were conducted. The three $\mathrm{pH}$ values were selected because they were in the three $\mathrm{pH}$ zones in Table 4 respectively. The elemental compositions of the samples determined by XPS were summarized in Table $\mathrm{S} 4 . \dagger$ The molecular formula of SMX is $\mathrm{C}_{10} \mathrm{H}_{11} \mathrm{~N}_{3} \mathrm{O}_{3} \mathrm{~S}$. Since the fresh carbon contained neither nitrogen $(\mathrm{N})$ nor sulfur $(\mathrm{S})$, the two elements detected by XPS could be safely attributed to SMX. The ratios of $\mathrm{N}$ to $\mathrm{S}$ were close to 3 , in accordance with the formula of SMX. Besides, the order in $\mathrm{N}$ and $\mathrm{S}$ contents was also in consistent with the order in SMX adsorption amounts (Fig. 5a).
An increase in the ratio of oxygen (O) to carbon (C) was observed after adsorption. This could also be attributed to the adsorption of SMX because the O/C ratio in SMX (0.3) was much higher than that in the fresh C-900 (0.028).

Deconvolution of the XPS spectra further revealed the types of heteroatoms (Fig. S5 $\dagger$ ). The peak positions in the N1s (Fig. S5a $\dagger$ ) and S2p (Fig. S5b $\dagger$ ) spectra were in line with those in previous reports, ${ }^{\mathbf{4 0}}$ verifying the uptake of SMX by the carbon. The oxygen-containing groups were presented by two peaks around 532 and $534 \mathrm{eV}$ (Fig. S5c $\dagger$ ), assigning to quinone type and/or $\mathrm{P}=\mathrm{O}$ groups $(\mathrm{O}-1)$ and phenol/ether type and/or $\mathrm{C}-\mathrm{O}-\mathrm{P}$ groups (O-2) respectively ${ }^{41}$ Deconvolution of the $\mathrm{P} 2 \mathrm{p}$ spectra (Fig. S5d $\dagger$ ) demonstrated two main signals around 132 and $134 \mathrm{eV}$, representing phosphorus in the carbon matrix $(\mathrm{P}-\mathrm{C})$ and $\mathrm{C}-\mathrm{O}-\mathrm{P}$ functionalities $(\mathrm{P}-\mathrm{O}) .{ }^{28,39}$ The relative amount of $\mathrm{O}-1$ and $\mathrm{O}-2$ groups changed after adsorption. On one hand, extra oxygen introduced through the adsorption of SMX triggered some change. On the other hand, the contribution of oxygencontaining groups in the carbon in the adsorption process may also make a difference. ${ }^{42}$ Similarly, the decrease in the relative amount of $\mathrm{P}-\mathrm{O}$ group after adsorption may also be attributed the contribution of oxygen-containing groups during SMX adsorption.

Raman spectra of the samples were shown in Fig. S6. $\dagger$ The patterns possessed D and $\mathrm{G}$ bands, respectively arising from the disordered carbon structures and the in-plane vibration mode. ${ }^{43}$ The positions of $\mathrm{D}$ and $\mathrm{G}$ bands were summarized in Table S5. $\dagger$ The bands shifted slightly to a higher frequency after adsorption for the spent samples at $\mathrm{pH} 2$ and 5. For the spent sample at pH 9, no obvious shift was observed. According to previous reports, ${ }^{44}$ an electron-donor causes a shift to lower frequency while an electron-acceptor induces an increase in frequency. Thus, the results here indicated that SMX mainly acted as an electron-acceptor and that the EDA interaction was relatively stronger at $\mathrm{pH} 2$ and 5 compared to that at $\mathrm{pH}$ 9, which supported the order of EDA interaction in Table 4.

\subsection{Regeneration}

It well known that the adsorption equilibrium is dynamic. Once the condition changes, a new equilibrium will tend to be established. For instance, the pseudo-equilibrium concentration of SMX in the bulk aqueous solution was found to be $2.1 \mathrm{mg} \mathrm{L}{ }^{-1}$ when $\mathrm{C}-900$ was employed to treat a $50 \mathrm{mg} \mathrm{L}^{-1}$ solution with an adsorbent dosage of $0.2 \mathrm{~g} \mathrm{~L}^{-1}$ at $30^{\circ} \mathrm{C}$. If the solution at equilibrium was replaced with the same amount of solution with a lower SMX concentration, desorption of SMX will certainly occur until a new equilibrium was established. According to the Le Chatelier's principle, the new equilibrium SMX concentration in the solution will be larger than zero but lower than $2.1 \mathrm{mg} \mathrm{L}^{-1}$. We conducted an additional experiment to address this issue by separating the spent carbon and add the same amount of deionized water. After shaking in a shaker bath for $48 \mathrm{~h}$, the bulk SMX concentration was measured to be $1.5 \mathrm{mg} \mathrm{L}^{-1}$, in consistent with the above discussion. From a practical viewpoint, the concentration of SMX in water should be very low after adsorption treatment to minimalize its 
detrimental effect to human health. Thus, water is not a suitable solvent for SMX desorption. Due to the much larger solubility of SMX in alcohol than in water, desorption of SMX is favored when alcohol was employed for regeneration. Similar results have been reported in previous works where it was found methanol or ethanol was efficient to desorb SMX while water was not. ${ }^{11,42,45}$ Thus, ethanol was used for the regeneration of C900 for potential applications. A slight decrease in the adsorption amount of SMX on the regenerated adsorbent was observed. After four adsorption cycles, C-900 could still afford $>90 \%$ of the initial adsorption amount (Fig. S7 $\dagger$ ), indicating its acceptable recyclability.

The maximum adsorption capacities of SMX over various carbon adsorbents were summarized in Table S6. $†$ C-900 in this work possessed a comparable or higher SMX adsorption capacity than most of these adsorbents in previous reports under similar experimental conditions. C-900 also had several economic and environmental benefits. From the cradle-to-grave scheme of the carbon (Fig. S1†), it can be found that low-cost natural precursor (sucrose) was used, and the preparation procedure was simplified through the in situ hydrothermal impregnation approach. The carbons in this work were only prepared in a laboratory scale and analytical grade reagents were utilized in the fabrications procedure. Based on the prices of sucrose (12 RMB/500 g) and $\mathrm{H}_{3} \mathrm{PO}_{4}(30 \mathrm{RMB} / 500 \mathrm{~g})$ as well as the carbon yield $(8.8 \%)$, the material cost of C-900 was estimated to be $1.6 \mathrm{RMB} / \mathrm{g}$. Besides, waste gas and water will be produced in the whole life cycle of the carbon (Fig. S1 $\dagger$ ). Adding the energy, labor and waste treatment cost will lead to a higher price, which seems not competitive compared to commercial activated carbons. However, much cheaper industrial grade reagents can be utilized for large scale production. Combined with its remarkable and regenerable adsorption ability, it should be considered as a promising candidate for efficient adsorptive removal of sulfamethoxazole.

\section{Conclusions}

A series of activated carbons denoted as C-500-C-900 were synthesized by hydrothermal impregnation of sucrose with $\mathrm{H}_{3} \mathrm{PO}_{4}$ and subsequent activation at $500-900{ }^{\circ} \mathrm{C}$. Through the in situ hydrothermal impregnation approach, the whole preparation process was simplified. The surface area of the resulting carbon tended to be promoted with an increase in the activation temperature, and the surface area of C-900 was boosted to 1929 $\mathrm{m}^{2} \mathrm{~g}^{-1}$, much higher than that of C-500 $\left(927 \mathrm{~m}^{2} \mathrm{~g}^{-1}\right)$. The carbons were employed for the adsorption removal of sulfamethoxazole in a batch mode, and the effects of contact time, adsorbent dosage, initial concentration, adsorption temperature and $\mathrm{pH}$ on SMX adsorption were studied. A good positive linear trend with a regression coefficient of 0.951 was observed between the Langmuir maximum adsorption capacities of SMX $\left(q_{\max }\right)$ and the carbon surface areas $\left(S_{\mathrm{BET}}\right)$, and C-900 performed best among the carbons with the highest $q_{\max }$ of $808.7 \mathrm{mg} \mathrm{g}^{-1}$, much higher than that of C-500 $\left(274.0 \mathrm{mg} \mathrm{g}^{-1}\right)$. The pseudosecond-order and the intra-particle diffusion model were used to analyze the adsorption kinetic data, and the results indicated that the presence of more mesopores in the carbon could favor the diffusion of SMX, leading to a faster adsorption rate. Adsorption thermodynamic results showed that the adsorption of SMX was a spontaneous exothermic process, with a standard enthalpy change of $-6.59 \mathrm{~kJ} \mathrm{~mol}^{-1}$ and a standard entropy change of $47.7 \mathrm{~J} \mathrm{~mol}^{-1} \mathrm{~K}^{-1}$. Furthermore, the plausible adsorption mechanism was proposed to be a combination of hydrophobic, electron donor-acceptor and electrostatic interactions with the assistance of XPS and Raman analysis. Regeneration tests showed that C-900 could still afford $>90 \%$ of the initial adsorption amount after four adsorption cycles through ethanol washing, indicating its acceptable recyclability.

\section{Conflicts of interest}

There are no conflicts of interest to declare.

\section{Acknowledgements}

Financial support from the National Natural Science Foundation of China [Grant No. 51638011, 51478314], the Science and Technology Plans of Tianjin [Grant No. 17PTSYJC00050, 17ZYPTJC00060] and China Postdoctoral Science Foundation [Grant No. 2018M641655] are gratefully acknowledged.

\section{References}

1 C. Ramprasad and L. Philip, Contributions of various processes to the removal of surfactants and personal care products in constructed wetland, Chem. Eng. J., 2018, 334, 322-333.

2 B. Jiang, A. Li, D. Cui, R. Cai, F. Ma and Y. Wang, Biodegradation and metabolic pathway of sulfamethoxazole by Pseudomonas psychrophila HA-4, a newly isolated cold-adapted sulfamethoxazole-degrading bacterium, Appl. Microbiol. Biotechnol., 2014, 98(10), 46714681.

3 W. Ben, Z. Qiang, C. Adams, H. Zhang and L. Chen, Simultaneous determination of sulfonamides, tetracyclines and tiamulin in swine wastewater by solid-phase extraction and liquid chromatography-mass spectrometry, $J$. Chromatogr. A, 2008, 1202(2), 173-180.

4 W. Ben, B. Zhu, X. Yuan, Y. Zhang, M. Yang and Z. Qiang, Occurrence, removal and risk of organic micropollutants in wastewater treatment plants across China: comparison of wastewater treatment processes, Water Res., 2018, 130, 3846.

5 M. Zbair, H. Ait Ahsaine and Z. Anfar, Porous carbon by microwave assisted pyrolysis: an effective and low-cost adsorbent for sulfamethoxazole adsorption and optimization using response surface methodology, $J$. Cleaner Prod., 2018, 202, 571-581.

$6 \mathrm{~F}$. Yu, Y. Li, S. Han and J. Ma, Adsorptive removal of antibiotics from aqueous solution using carbon materials, Chemosphere, 2016, 153, 365-385.

7 C. B. Vidal, M. Seredych, E. Rodriguez-Castellon, R. F. Nascimento and T. J. Bandosz, Effect of nanoporous 
carbon surface chemistry on the removal of endocrine disruptors from water phase, J. Colloid Interface Sci., 2015, 449, 180-191.

8 L. Ji, F. Liu, Z. Xu, S. Zheng and D. Zhu, Adsorption of Pharmaceutical Antibiotics on Template-Synthesized Ordered Micro- and Mesoporous Carbons, Environ. Sci. Technol., 2010, 44(8), 3116-3122.

9 H. Chen, B. Gao and H. Li, Removal of sulfamethoxazole and ciprofloxacin from aqueous solutions by graphene oxide, $J$. Hazard. Mater., 2015, 282, 201-207.

10 L. Ji, W. Chen, S. Zheng, Z. Xu and D. Zhu, Adsorption of sulfonamide antibiotics to multiwalled carbon nanotubes, Langmuir, 2009, 25(19), 11608-11613.

11 I. Ahmed, B. N. Bhadra, H. J. Lee and S. H. Jhung, Metalorganic framework-derived carbons: Preparation from ZIF8 and application in the adsorptive removal of sulfamethoxazole from water, Catal. Today, 2018, 301, 90-97.

$12 \mathrm{X}$. Li, H. Yuan, X. Quan, S. Chen and S. You, Effective adsorption of sulfamethoxazole, bisphenol A and methyl orange on nanoporous carbon derived from metal-organic frameworks, J. Environ. Sci., 2017, 63, 250-259.

13 M.-M. Titirici, R. J. White, C. Falco and M. Sevilla, Black perspectives for a green future: hydrothermal carbons for environment protection and energy storage, Energy Environ. Sci., 2012, 5(5), 6796-6822.

14 X. Zhu, Y. Liu, F. Qian, C. Zhou, S. Zhang and J. Chen, Role of Hydrochar Properties on the Porosity of Hydrochar-based Porous Carbon for Their Sustainable Application, ACS Sustainable Chem. Eng., 2015, 3(5), 833-840.

15 Y. Shi, X. Zhang and G. Liu, Activated Carbons Derived from Hydrothermally Carbonized Sucrose: Remarkable Adsorbents for Adsorptive Desulfurization, ACS Sustainable Chem. Eng., 2015, 3(9), 2237-2246.

16 M. Wisniewski, A. Pacholczyk, A. P. Terzyk and G. Rychlicki, New phosphorus-containing spherical carbon adsorbents as promising materials in drug adsorption and release, $J$. Colloid Interface Sci., 2011, 354(2), 891-894.

17 A. J. Romero-Anaya, M. A. Lillo-Ródenas, C. Salinas-Martínez de Lecea and A. Linares-Solano, Hydrothermal and conventional $\mathrm{H}_{3} \mathrm{PO}_{4}$ activation of two natural bio-fibers, Carbon, 2012, 50(9), 3158-3169.

18 Z.-b. Zhang, Z.-w. Zhou, X.-h. Cao, Y.-h. Liu, G.-x. Xiong and P. Liang, Removal of uranium(VI) from aqueous solutions by new phosphorus-containing carbon spheres synthesized via one-step hydrothermal carbonization of glucose in the presence of phosphoric acid, J. Radioanal. Nucl. Chem., 2013, 299(3), 1479-1487.

19 W. Lei, Y.-P. Deng, G. Li, Z. P. Cano, X. Wang, D. Luo, Y. Liu, D. Wang and Z. Chen, Two-Dimensional Phosphorus-Doped Carbon Nanosheets with Tunable Porosity for Oxygen Reactions in Zinc-Air Batteries, ACS Catal., 2018, 8(3), 2464-2472.

20 K. G. Gorbovskiy, A. I. Kazakov, A. M. Norov and A. I. Mikhaylichenko, Effect of Impurities on Thermal Decomposition Kinetics of Mineral Fertilizers Based on $\left(\mathrm{NH}_{4}\right)_{2} \mathrm{HPO}_{4}$ in Self-Generated Atmosphere, Russ. J. Appl. Chem., 2018, 91(7), 1057-1067.
21 A. J. Romero-Anaya, M. Ouzzine, M. A. Lillo-Ródenas and A. Linares-Solano, Spherical carbons: synthesis, characterization and activation processes, Carbon, 2014, 68, 296-307.

22 P. Llewellyn, F. Rodriquez-Reinoso, J. Rouqerol and N. Seaton, Is the BET equation applicable to microporous adsorbents?, Stud. Surf. Sci. Catal., 2007, 160, 49.

23 P. Yaseneva, C. F. Marti, E. Palomares, X. Fan, T. Morgan, P. S. Perez, M. Ronning, F. Huang, T. Yuranova, L. KiwiMinsker, S. Derrouiche and A. A. Lapkin, Efficient reduction of bromates using carbon nanofibre supported catalysts: Experimental and a comparative life cycle assessment study, Chem. Eng. J., 2014, 248, 230-241.

24 N. Arena, J. Lee and R. Clift, Life Cycle Assessment of activated carbon production from coconut shells, J. Cleaner Prod., 2016, 125, 68-77.

25 Y. K. Kim, J. H. Park and J. W. Lee, Facile nano-templated $\mathrm{CO}_{2}$ conversion into highly interconnected hierarchical porous carbon for high-performance supercapacitor electrodes, Carbon, 2018, 126, 215-224.

26 Y. Shi, G. Liu, L. Wang and X. Zhang, Efficient adsorptive removal of dibenzothiophene from model fuel over heteroatom-doped porous carbons by carbonization of an organic salt, Chem. Eng. J., 2015, 259, 771-778.

27 M. Sevilla, A. B. Fuertes and R. Mokaya, High density hydrogen storage in superactivated carbons from hydrothermally carbonized renewable organic materials, Energy Environ. Sci., 2011, 4(4), 1400-1410.

28 S. Chen, J. Wang, Z. Wu, Q. Deng, W. Tu, G. Dai, Z. Zeng and S. Deng, Enhanced $\mathrm{Cr}(\mathrm{VI})$ removal by polyethylenimine- and phosphorus-codoped hierarchical porous carbons, J. Colloid Interface Sci., 2018, 523, 110-120.

29 H. N. Tran, S. J. You, A. Hosseini-Bandegharaei and H. P. Chao, Mistakes and inconsistencies regarding adsorption of contaminants from aqueous solutions: a critical review, Water Res., 2017, 120, 88-116.

30 K. L. Tan and B. H. Hameed, Insight into the adsorption kinetics models for the removal of contaminants from aqueous solutions, J. Taiwan Inst. Chem. Eng., 2017, 74, 2548.

31 N. Ouasfi, S. Bouzekri, M. Zbair, H. Ait Ahsaine, S. Bakkas, M. Bensitel and L. Khamliche, Carbonaceous material prepared by ultrasonic assisted pyrolysis from algae (Bifurcaria bifurcata): response surface modeling of aspirin removal, Surf. Interfaces, 2019, 14, 61-71.

32 M. Kah, G. Sigmund, F. Xiao and T. Hofmann, Sorption of ionizable and ionic organic compounds to biochar, activated carbon and other carbonaceous materials, Water Res., 2017, 124, 673-692.

33 M. D. Teli and G. T. Nadathur, Adsorptive removal of acid yellow 17 (an anionic dye) from water by novel ionene chloride modified electrospun silica nanofibres, J. Environ. Chem. Eng., 2018, 6(6), 7257-7272.

34 J. Dai, A. Xie, R. Zhang, W. Ge, Z. Chang, S. Tian, C. Li and Y. Yan, Scalable preparation of hierarchical porous carbon from lignin for highly efficient adsorptive removal of sulfamethazine antibiotic, J. Mol. Liq., 2018, 256, 203-212. 
35 M. B. Ahmed, J. L. Zhou, H. H. Ngo, W. Guo, M. A. H. Johir and K. Sornalingam, Single and competitive sorption properties and mechanism of functionalized biochar for removing sulfonamide antibiotics from water, Chem. Eng. J., 2017, 311, 348-358.

36 L. Nielsen, M. J. Biggs, W. Skinner and T. J. Bandosz, The effects of activated carbon surface features on the reactive adsorption of carbamazepine and sulfamethoxazole, Carbon, 2014, 80, 419-432.

37 P. A. Quinlivan, L. Li and D. R. Knappe, Effects of activated carbon characteristics on the simultaneous adsorption of aqueous organic micropollutants and natural organic matter, Water Res., 2005, 39(8), 1663-1673.

38 Q. Chen, J. Zheng, J. Xu, Z. Dang and L. Zhang, Insights into sulfamethazine adsorption interfacial interaction mechanism on mesoporous cellulose biochar: coupling DFT/FOT simulations with experiments, Chem. Eng. J., 2019, 356, 341-349.

39 R. Gao, L. Pan, J. Lu, J. Xu, X. Zhang, L. Wang and J.-J. Zou, Phosphorus-Doped and Lattice-Defective Carbon as Metallike Catalyst for the Selective Hydrogenation of Nitroarenes, ChemCatChem, 2017, 9(22), 4287-4294.
40 X.-Y. Xu, C. Chu, H. Fu, X.-D. Du, P. Wang, W. Zheng and C.-C. Wang, Light-responsive UiO-66- $-\mathrm{NH}_{2} / \mathrm{Ag}_{3} \mathrm{PO}_{4}$ MOFnanoparticle composites for the capture and release of sulfamethoxazole, Chem. Eng. J., 2018, 350, 436-444.

41 W. Ma, L. Xie, L. Dai, G. Sun, J. Chen, F. Su, Y. Cao, H. Lei, Q. Kong and C.-M. Chen, Influence of phosphorus doping on surface chemistry and capacitive behaviors of porous carbon electrode, Electrochim. Acta, 2018, 266, 420-430.

42 R. Li, Y. Zhang, W. Chu, Z. Chen and J. Wang, Adsorptive removal of antibiotics from water using peanut shells from agricultural waste, $R S C$ Adv., 2018, 8(24), 13546-13555.

43 H. Javed, D. X. Luong, C.-G. Lee, D. Zhang, J. M. Tour and P. J. J. Alvarez, Efficient removal of bisphenol-A by ultrahigh surface area porous activated carbon derived from asphalt, Carbon, 2018, 140, 441-448.

44 B. Das, R. Voggu, C. S. Rout and C. N. R. Rao, Changes in the electronic structure and properties of graphene induced by molecular charge-transfer, Chem. Commun., 2008, 41, 5155-5157.

45 Q. Song, H. Wang, B. Yang, F. Wang and X. Sun, A novel adsorbent of Ag-FMWCNTs for the removal of SMX from aqueous solution, $R S C A d v$., 2016, 6(79), 75855-75861. 